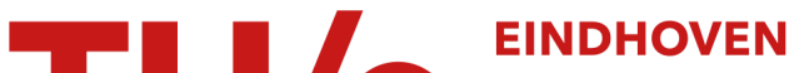 \\ UNIVERSITY OF \\ TECHNOLOGY
}

\section{InP photonic circuits using generic integration}

Citation for published version (APA):

Williams, K. A., Bente, E. A. J. M., Heiss, D., Jiao, Y., Lawniczuk, K., Leijtens, X. J. M., Tol, van der, J. J. G. M., \& Smit, M. K. (2015). InP photonic circuits using generic integration. Photonics Research, 3(5), B60-B68.

https://doi.org/10.1364/PRJ.3.000B60

DOI:

10.1364/PRJ.3.000B60

Document status and date:

Published: 01/01/2015

\section{Document Version:}

Publisher's PDF, also known as Version of Record (includes final page, issue and volume numbers)

\section{Please check the document version of this publication:}

- A submitted manuscript is the version of the article upon submission and before peer-review. There can be important differences between the submitted version and the official published version of record. People interested in the research are advised to contact the author for the final version of the publication, or visit the $\mathrm{DOI}$ to the publisher's website.

- The final author version and the galley proof are versions of the publication after peer review.

- The final published version features the final layout of the paper including the volume, issue and page numbers.

Link to publication

\section{General rights}

Copyright and moral rights for the publications made accessible in the public portal are retained by the authors and/or other copyright owners and it is a condition of accessing publications that users recognise and abide by the legal requirements associated with these rights.

- Users may download and print one copy of any publication from the public portal for the purpose of private study or research.

- You may not further distribute the material or use it for any profit-making activity or commercial gain

- You may freely distribute the URL identifying the publication in the public portal.

If the publication is distributed under the terms of Article 25fa of the Dutch Copyright Act, indicated by the "Taverne" license above, please follow below link for the End User Agreement:

www.tue.nl/taverne

Take down policy

If you believe that this document breaches copyright please contact us at:

openaccess@tue.nl

providing details and we will investigate your claim. 


\title{
InP photonic circuits using generic integration [Invited]
}

\author{
K. A. Williams, ${ }^{*}$ E. A. J. M. Bente, D. Heiss, Y. Jiao, K. Lawniczuk, X. J. M. Leijtens, \\ J. J. G. M. van der Tol, and M. K. Smit \\ COBRA Research Institute, Eindhoven University of Technology, P.O. Box 513, Eindhoven 5600MB, The Netherlands \\ *Corresponding author: k.a.williams@tue.nl
}

Received May 18, 2015; revised July 7, 2015; accepted July 16, 2015;

posted July 23, 2015 (Doc. ID 241319); published August 28, 2015

InP integrated photonics has become a critical enabler for modern telecommunications, and is poised to revolutionize data communications, precision metrology, spectrometry, and imaging. The possibility to integrate high-performance amplifiers, lasers, modulators, and detectors in combination with interferometers within one chip is enabling game-changing performance advances, energy savings, and cost reductions. Generic integration accelerates progress through the separation of applications from a common technology development. In this paper, we review the current status in InP integrated photonics and the efforts to integrate the next generation of high-performance functionality on a common substrate using the generic methodology. (C) 2015 Chinese Laser Press

OCIS codes: $\quad$ (250.0250) Optoelectronics; (250.5300) Photonic integrated circuits.

http://dx.doi.org/10.1364/PRJ.3.000B60

\section{INTRODUCTION}

InP-based quaternary alloys have played an enabling role in the critically important 1.1-1.6 $\mu \mathrm{m}$ spectral window for fiber-optic systems. InGaAsP and InGaAlAs quaternary alloys specifically have allowed considerable flexibility in terms of the range of engineered bandgaps and refractive indices that can be achieved on high-quality, low-defect-density InP substrates. The ability to include a wide range of optical waveguide devices with different direct bandgaps on the same substrate has led to a powerful class of InP integrated photonic circuits with both passive components such as optical splitters, filters, multiplexers, and combiners and active components such as optical amplifiers, lasers, modulators, and detectors.

Integrated circuit technology improves circuit-level performance by removing assembly complexity and variability. As the technology matures, increasing numbers of component-level interconnections are made at the wafer scale. This enables sustained increases in the functionality, performance, and reliability of circuits, while reducing their size, power, and cost [1]. As technology matures, integrated circuit products outperform equivalent combinations of discrete components at the functional level. Digital coherent transceivers are a striking example [2]. Such complexity was impractical until integration technologies enabled a new class of $100+\mathrm{Gb} / \mathrm{s}$ communications products and technologies. New concepts for cost-effective, high-connectivity optical packet switching circuits are similarly inconceivable without extensive integration [3].

Performance advances have been enabled through sustained technology developments for InP-based epitaxial and fabrication processes as well as device design innovations. Killer defect densities have been driven down to levels comparable with silicon CMOS in the early 1990s [4] with Infinera reporting random killer defect densities in the range of 0.5 to $1.25 \mathrm{~cm}^{-2}$ and functional yields as high as $70 \%$ for 440 -element circuits [5]. Improvements in reliability are becoming a key advantage for InP integrated photonics technology as performance yield is determined increasingly by packaging and assembly. Strict design methodologies and tightly controlled processes have been essential. Vertically integrated corporations such as Infinera [4] and open-access platforms such as JePPIX [6] have adopted a methodology for InP-based photonic integrated circuits (PICs) that is similar to the CMOS electronic integrated circuits approach: designers are given a fixed tool set so that fabs can deliver manufacturable, cost-effective devices. For an open-access platform, this takes the form of a fab-specific process design kit (PDK) $[7,8]$.

In this paper, we review the recent progress in InP integrated photonic circuits exploiting generic methodologies. In Section 2 we highlight the current status of the powerful generic InP integration platform, which already enables laserand amplifier-based integrated circuits in the same chip. In Section 3 we address the new component innovations that are expected to feed into future platform releases. In Section 4, we highlight longer-term research that enables further miniaturization using our InP membrane on silicon (IMOS) platform. In Section 5 we address opportunities for future platform convergence with silicon electronics and low-loss dielectric-waveguide technologies.

\section{GENERIC INTEGRATION PLATFORM}

The power of the InP generic integration platform derives from the innate single-chip integration of lasers, modulators, amplifiers, and detectors [6]. Originally the generic integration methodology was conceived as an academic concept in the ePIXnet network of excellence to enable many photonics applications to leverage the same efforts in technology development. This methodology has been transferred to the industry through the EuroPIC and Paradigm projects [9]. More than 250 custom chips have now been produced at Oclaro, Fraunhofer 
Table 1. Generic Integrated Photonics: Current Capability for InP Open Access Technology [10]

\begin{tabular}{lcccc}
\hline Building Block & Fraunhofer & Oclaro & Smart \\
\hline Waveguide (strong and weak) & $\checkmark$ & $\checkmark$ & $\checkmark$ & $2-3$ dB for low-loss guides \\
Transition elements (active/passive and strong/weak) & $\checkmark$ & $\checkmark$ & Enabler for PIC design \\
Waveguide crossing & $\checkmark$ & $\checkmark$ & Complex optical functions \\
Electrical isolation & $\checkmark$ & $\checkmark$ & High-density integration \\
Curved waveguides & $\checkmark$ & $\checkmark$ & High-density integration \\
Metal waveguides and lines over optical guides & $\checkmark$ & $\checkmark$ & For low-loss fiber coupling \\
Spot size converter & $\checkmark$ & $\checkmark$ & Low-energy tuning \\
Current-based phase shifter & & $\checkmark$ & Circuit control \\
Thermo-optic phase shifter & $\checkmark$ & $\checkmark$ & High-speed data \\
Voltage-based phase modulator & & $\checkmark$ & High-speed data \\
PIN photodiode & $\checkmark$ & $\checkmark$ & High-speed data \\
Balanced PIN photodiode & $\checkmark$ & $\checkmark$ & Interferometry \\
Couplers and splitters & $\checkmark$ & $\checkmark$ & Critical enabler for lasers \\
Optical amplifiers & & $\checkmark$ & Advanced lasers \\
Tunable Bragg gratings & & $\checkmark$ & & \\
\hline
\end{tabular}

HHI, and Smart Photonics using JePPIX Open Access brokering.

Powerful PDKs embedded in commercial software tools are enabling this ramp-up in the number of realized circuits. The PDKs provide designers with sufficient information to create their circuits, and the simulation tools are increasingly coupled to the fab-specific performance for the industryproduced chips. The libraries are hierarchically built on the concept of basic building blocks [6]. The most basic elements in the circuit include phase modulators, amplifiers, and gratings. Combinations of these can be used to create composite building blocks such as lasers, interferometric modulators, and multiplexers. The range of building blocks continues to expand, and Table 1 provides an overview of the capability offered by JePPIX today [8,10].

High-performance active devices are the unique selling point for InP integrated photonics: optical amplifiers can operate with tens of milliwatts; single frequency, pulsed, and tunable lasers are readily integrated; detectors and modulators operate with bandwidths from DC $-40 \mathrm{GHz}$. The performance of passive components is also increasing to enable tighter specification. In this section we review a number of highlights from the most promising application sectors exploiting this approach.

Advanced de/modulation techniques are set to increase in sophistication with innovations in integration. Powerful new optical techniques may be able to perform certain signal processing tasks more efficiently in the optical domain [11]. A recent circuit for optical demodulation of $40 \mathrm{~Gb} / \mathrm{s}$ differential phase-shift keyed signals has been proposed, implemented, and assessed. The circuit consists of a onebit-delay interferometer and a semiconductor optical amplifier (SOA) where the constructive and destructive interferometer outputs counterpropagate in a gain saturated amplifier to create a reshaping effect. A net improvement in the signal-tonoise ratio is observed when the circuit is fed with a noisy input signal.

Fiber-to-the-home consumer services are expected to drive higher and higher line-rates. This becomes feasible with multiwavelength transmitters. The example circuit for central office implementation is shown in Fig. 1. This is realized by integrating an array of eight distributed Bragg reflector (DBR) lasers with an array of four Mach-Zehnder modulators.
An arrayed waveguide grating is used to multiplex the optical channels into a common optical output waveguide [12]. The four continuous wave pilot tones are used for remotely modulated upstream data, and the locally modulated channels enable the downstream link. Operating powers of $+4 \mathrm{dBm}$ are achieved into the fiber with a data rate of $12.5 \mathrm{~Gb} / \mathrm{s}$ per transmission channel.

Spectroscopic imaging techniques used in the biosciences and materials analysis are set to benefit from precision control of picosecond and femtosecond optical pulse sources. Integrated pulse sources have so far been plagued by waveguide dispersion, and it has proved very challenging to create high-quality symmetry pulses. This has motivated techniques to electronically program the chirp characteristics of photonic pulse sources on chip. Figure 2 shows one such chip, which has been realized and demonstrated for highly chirped optical pulses [13]. The device integrates a 20-channel arrayed waveguide grating with 20 phase modulators and 20 SOAs on a single chip with area $6 \mathrm{~mm} \times 6 \mathrm{~mm}$. Asymmetric pulses with durations of $3.0 \mathrm{ps}(6.6 \mathrm{ps})$ at the $3 \mathrm{~dB}(6 \mathrm{~dB})$ widths are compressed to create more symmetric pulses with optical pulsewidths of $2.2 \mathrm{ps}$ ( $2.9 \mathrm{ps}$ ), respectively. This offers compatibility with innovative new picosecond pulse sources that are being devised on the same generic platform [14-17]. Record bandwidths are now also being achieved using

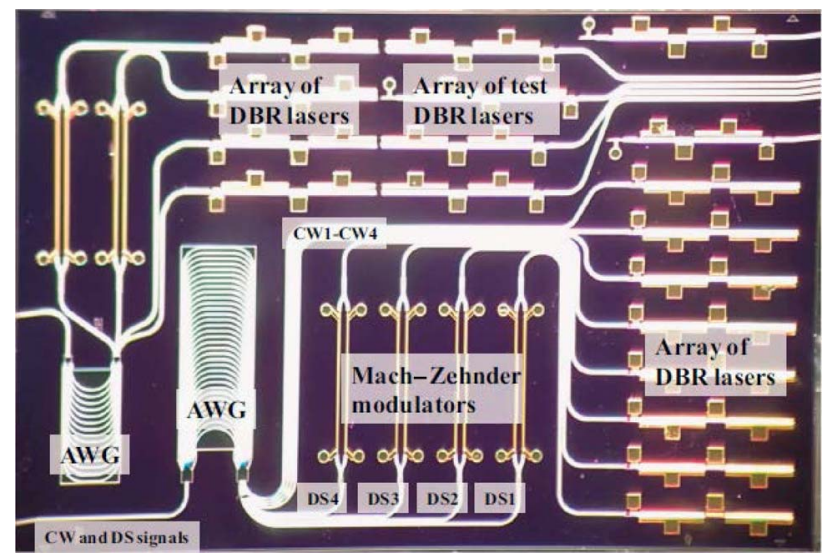

Fig. 1. Multiwavelength transmitter created from Bragg reflectors, optical amplifiers, phase modulators, and interferometers [12]. 




Fig. 2. Pulse compressor using on-chip gain and phase control to manipulate individual Fourier lines in the optical pulse spectrum [13].

ring-resonator-based mode-locked lasers on such a platform [18]. The same combination of building blocks may also be used to create widely tunable lasers at $1.7 \mu \mathrm{m}$ [19]. An appropriate choice of the first epitaxial wafer combined with the same process technology enables a broad range of laser designs operating with a range in emission wavelengths.

Structural health monitoring systems using distributed fiber sensors can exploit precision wavelength metering in sophisticated photonic readouts. Integration technology is enabling cost-effective, stable interferometry for such applications. A picometer precision wavelength meter has been implemented through the use of a $1 \times 8$ arrayed waveguide grating in combination with an array of photodetectors [19]. The free spectral range is designed to be $50 \mathrm{~nm}$, but a resolution of several orders of magnitude below the channel spacing is achieved through a simultaneous analysis of more than one channel. The sensitivity of the system is tested by using the passband roll-off of two adjacent arrayed waveguide channels. In the demonstration experiments, a laser wavelength discretely tuned in steps of $5 \mathrm{pm}$ in the range between two passband peaks shows that wavelength steps of a few pm can be clearly distinguished with a measured standard deviation of $0.8 \mathrm{pm}$.

Data center networks are anticipated to require packetcompliant switch fabrics with high capacity and scalable connectivity. The first, to the best of our knowledge, monolithically integrated InGaAsP/InP active-passive $8 \times 8$ cross connect able to route arbitrary combinations of wavelengths to fiber connections has been fabricated using the generic process technology [3]. This is shown in Fig. 3. The selection functionalities in the space and wavelength domains are implemented simultaneously on a single chip using a circuit comprising SOA gates, splitters, and cyclic wavelength routers. Eight broadband inputs connect to an array of eight $1 \times 8$ broadband space selection switches. Wavelength domain selection is subsequently performed with an array of eight $8 \times 8$ gated cyclic routers. The on-chip fan-outs and fan-ins are implemented to wire up the 136 SOA gates and eight cyclic routers within a chip area of $14.6 \mathrm{~mm} \times 6.7 \mathrm{~mm}$. The circuit has been demonstrated for $160 \mathrm{~Gb} / \mathrm{s}$ wavelength multiplexed throughputs under automated packet routing control [20]. Combinations of Mach-Zehnder interferometers and SOA gates have also been implemented to explore energyefficient data routing [21] using generic circuits produced

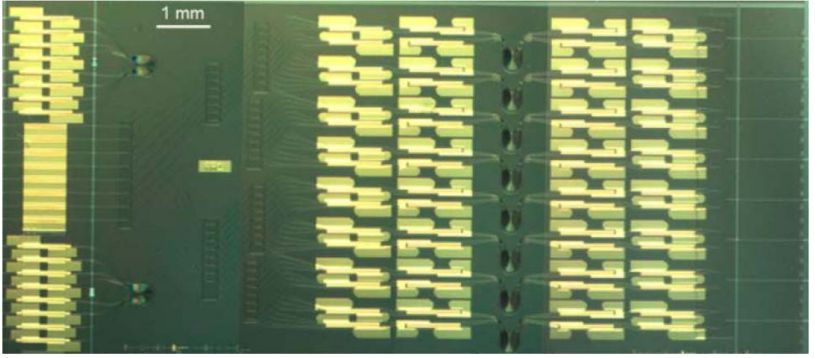

Fig. 3. Optical switching matrix connecting eight wavelength multiplexed inputs to eight outputs. The connections for each wavelength are electronically programmable with nanosecond reconfiguration times. [3].

on multiproject wafer runs. While these achievements are impressive in their own right, there is continued pressure to enhance circuit-level complexity and performance. This is met through sustained component-level innovation. In Section 3 we highlight research activities that may become available as new basic and composite building blocks for future platform releases.

\section{PHOTONIC DEVICE RESEARCH}

Innovations in circuit performance become feasible through ruggedized building blocks that are able to interconnect without adversely affecting each other. A clear focus on reducing loss and parasitic interaction is critical to performance enhancement. Here we review advances in key components for reducing loss, imperfections, parasitic reflections, and leakage. We address the enhancement of circuit-level functions for splitters, polarization devices, microbends, and de/multiplexers.

\section{A. Ultra-Low Reflection Splitters}

The parasitic reflections at interfaces between components and waveguides can occur due to abrupt changes in optical cross-sectional areas leading to a detrimental effect on circuit-level performance. This is particularly true for multimode interference (MMI) devices, which are commonly used to image one or more inputs to one or more output waveguides for splitting and filtering functions. In splitters, the abrupt transitions have lead to backreflected powers of order -10 to $-15 \mathrm{~dB}$, and this can be a cause of gain ripple in amplifiers and mode instabilities in many important classes of lasers. Recent work has reduced parasitic reflection to below $-35 \mathrm{~dB}$ by optimizing the waveguide geometry [22]. In the example shown in Fig. 4, all sidewalls of the multimode section of the $1 \times 2$ optical power splitter have been reduced to only those essential for the imaging. The access waveguides are defined through an engineered tapering of the deep-etch trench at the left input and right output waveguides. The

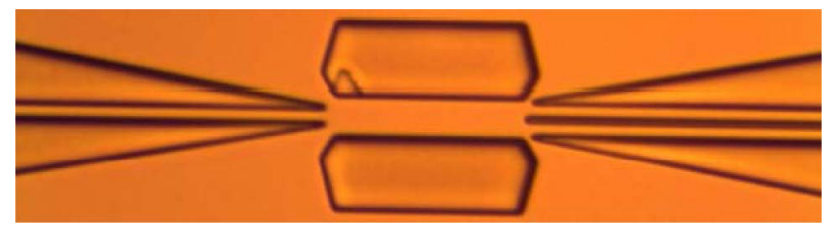

Fig. 4. Low reflection splitters: photograph of a $1 \times 2$ MMI splitter [22]. 
interfaces between the single and multimode regions are optimized through tapered features to minimize reflection and loss at each of the interfaces.

\section{B. Fabrication-Tolerant Polarization Converters}

Controlling the polarization within integrated circuits ameliorates the effect of variations in the input state of polarization. Sufficient levels of control enable the exploitation of polarization states to double communications capacity. Integrated polarization devices proposed to date have suffered from tight fabrication tolerances. This arises from two main effects: first, the difficulty in maintaining the polarization angle of the modes close enough to the optimal $45^{\circ}$ condition, and, second, the ability to control the beat length between the TE and TM modes (waveguide birefringence). The integrated polarization rotator is shown in Fig. 5. The electromagnetic boundary conditions rotate the polarization of the modes. For the correct length of slanted guide, an input TE mode equally excites the two rotated orthogonal modes at the output [23]. The precise length determines the power splitting. In common with many other designs, this is dependent on waveguide critical dimension variability between the designed waveguide width and the produced waveguide width. This can be of order $100 \mathrm{~nm}$ for today's InP process flows.

Recently a double-section polarization converter structure has been proposed and validated, and has been able to correct for critical dimension variation. The two-section polarization converter concept consists of two right trapezoidal sections with the wet-etched angled sidewalls to cancel the dominant source of variability [23-25]. The measured conversion efficiency of the one-section converters is higher than $96 \%$ in the $\mathrm{C}$ band, but the critical dimension needs to be controlled to within $\pm 60 \mathrm{~nm}$ of the design value. The two-section polarization converter gives an efficiency of polarization conversion above $99 \%$ over the $\mathrm{C}$ band, and, importantly, the critical dimension variability is relaxed to $\pm 130 \mathrm{~nm}$. The loss of the one-section converters is below $0.5 \mathrm{~dB}$, and the loss of the two-section converters is about $0.6 \mathrm{~dB}$.

\section{Microbends}

Curved waveguides offer the most flexible waveguide routing with an arbitrary change in angle and modest excess loss [26].

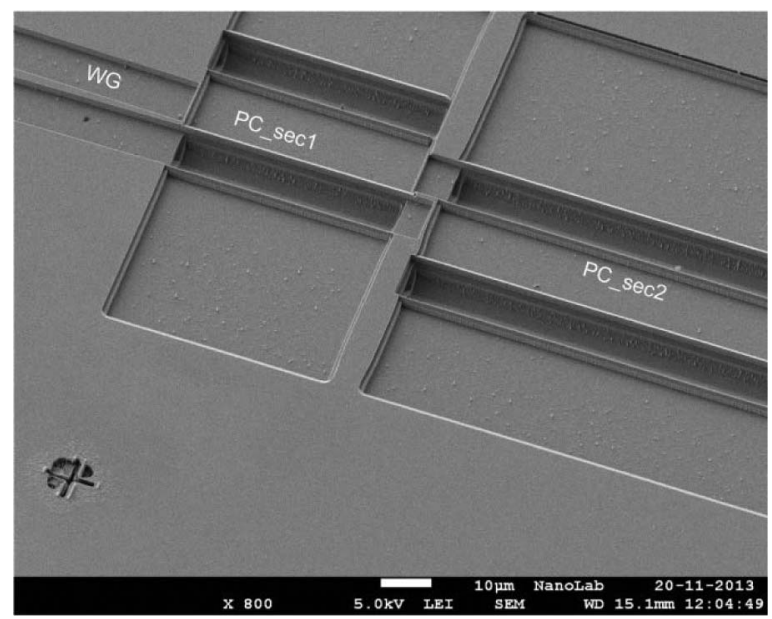

Fig. 5. Fabrication-tolerant two-section integrated polarization converter.

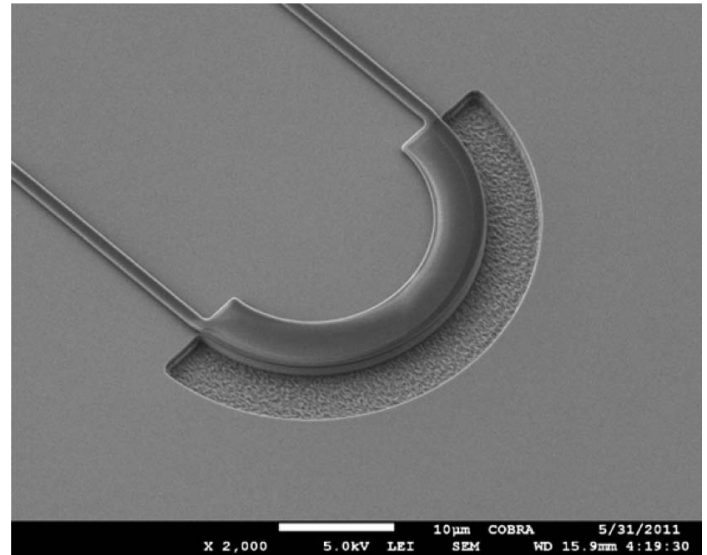

Fig. 6. Whispering gallery regime microbend with $20 \mu \mathrm{m}$ radius as proposed in [28].

Weak ridge waveguides enable low-loss bends of the order of hundreds of micrometers, but stronger, deep-etched waveguides with air or polymer sidewall claddings are needed to enable smaller radii of curvature. Designers strive to pack increasing numbers of components and functions into one InP chip, but as the radius is reduced, careful attention needs to be paid to mode matching between components, straight waveguides, and curved waveguides. Critical dimension control, sidewall angle, polarization rotation, and loss all become important.

Recently, whispering gallery regime microbends have been proposed to meet the critical dimension variation requirement and achieve low-loss routing in microbends. Figure 6 shows a recently fabricated $20 \mu \mathrm{m}$ radius microbend. The structure has been implemented with a deeper than usual etch depth and is used across a large scale of $16 \times 16$ switching matrices [27]. Here the critical design dimensions are relaxed by using a multimode waveguide in the bend structure. The optical mode hugs the outer radius as it propagates around the bend, while the inner radius is within the caustic radius and has minimum impact on the optical field. The critical offsetting dimensions between the input, output, and bent waveguides are defined in one mask layer, providing a fabrication-tolerant design. Simulations show that bend radii of down to even a few micrometers may be feasible. Microbend waveguide width variations of $100 \mathrm{~nm}$ lead to loss variations of only $0.01 \mathrm{~dB}$ in the whispering gallery regime [28]. Losses of only $0.2 \mathrm{~dB} / 180^{\circ}$ have been estimated from fabricated circuits and simulations alike [27,28]. By controlling the sidewall verticality, polarization conversion of down to $-25 \mathrm{~dB} / 180^{\circ}$ is predicted [28]. Circuit-level assessments show no measurable performance degradation through polarization rotation [27].

\section{Ultra-Low-Loss Waveguides}

Losses in InP photonic waveguides are dominated by scattering loss and absorption. As process control improves, the p-doped cladding becomes the most important contribution to loss through inter-valence band absorption. The n-type absorption is less significant and can be modeled by accounting for scattering introduced through electron-phonon and electron-ionized impurity interaction [29]. Low p-doped epitaxy has been assessed with loss-sensitive ring resonators 


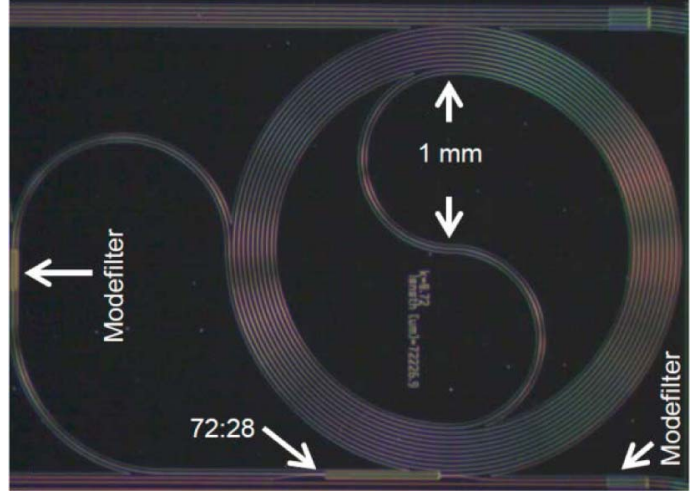

Fig. 7. Ultra-low-loss waveguides.

as shown in Fig. 7. Resonators with $80 \mathrm{~mm}$ long spiral delay lines are out-coupled via an imbalanced MMI coupler. The spiral loss is greatly reduced by using low p-doped, shallowly etched waveguides with millimeter bending radii. The measured propagation loss is only $0.3 \mathrm{~dB} / \mathrm{cm}$ for the wavelength range from 1450 to $1650 \mathrm{~nm}$. The ridge waveguide has a cross-sectional area of $2.5 \mu \mathrm{m} \times 0.6 \mu \mathrm{m}$ and a bend radius of $1 \mathrm{~mm}$. Equivalent waveguides with p-doped contact layers showed losses of order $2.0 \mathrm{~dB} / \mathrm{cm}$, highlighting an important route to circuit-level loss reductions.

\section{E. De/Multiplexers}

The arrayed waveguide grating has become a critical enabler in modern wavelength multiplexed communications. Extensive work in the miniaturization and passband spectral shaping has already been performed, but until recently, a number of key metrics have proved challenging to optimize. There has been a stark choice between fast, imprecise design tools and accurate but numerically intensive beam propagation solvers. A new analytical model has now been devised that calculates and uses realistic $1 \mathrm{D}$ mode field profiles to more accurately simulate the star coupler response [30]. This enables accurate de/multiplexer models to be created for circuit simulators. The inclusion of a MMI device model at the inputs also enables the accurate simulation of passband flattened arrayed waveguide gratings. The passband is calculated for each mode, and so the calculation time scales linearly with the number of input modes. An analytical simulation takes $4.6 \mathrm{~s}$ to simulate 2000 points and three modes, while a beam propagation method simulation takes $8.5 \mathrm{~h}$ for 200 points.

The transmission losses for the arrayed waveguide gratings are known to depend directly on the width of the gaps between the arrayed waveguides. This ultimately is limited by the critical dimension size achievable in the waveguide lithography. The requirement for precision gaps over a wide field of view mandates the use of deep UV lithography, and this brings with it the added potential advantages of reduced edge roughness for reduced waveguide loss and enhanced reproducibility for center wavelength reproducibility. Figure 8 shows a scanning electron microscope image for a recently fabricated arrayed waveguide grating. The $100 \mathrm{~nm}$ gaps between the $1.5 \mu \mathrm{m}$ wide deep-etch waveguide gratings enable a loss of $1.0 \mathrm{~dB}$. This compares favorably with the $3.0 \mathrm{~dB}$ losses that are typically achievable for InP arrayed waveguide gratings fabricated with gaps of $500 \mathrm{~nm}$.

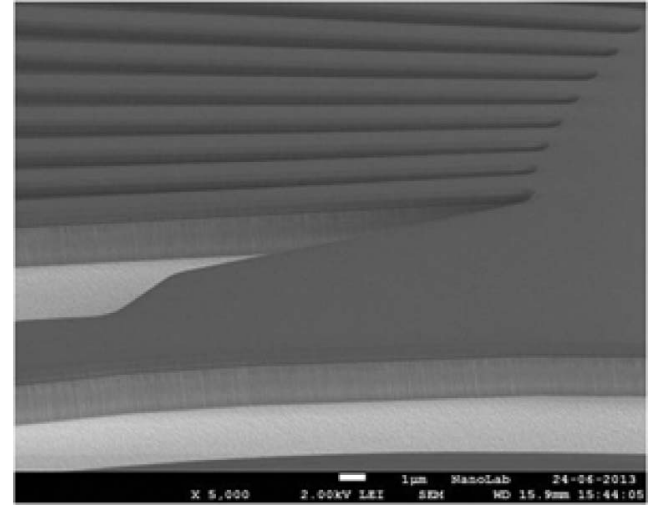

Fig. 8. Arrayed waveguide grating enabled through deep UV lithography.

\section{F. Intra-Chip Cavities}

On-chip broadband reflectors provide an important opportunity for creating lasers, cavities, and interferometers within the chip while avoiding wavelength selectivity or precision facet placement. This provides mask-defined precision for cavity lengths and a controllable reflectivity that is not determined by facet-cleaving and facet-coating steps. A new class of MMI couplers has been designed and demonstrated to act as on-chip mirrors. Partial out-coupling has been demonstrated as a two-port device with transmission and reflection, along with compact mirrors with full reflection in a one-port structure [31]. Measurements show losses as low as $0.4 \mathrm{~dB}$ for one-port devices and as low as $1.4 \mathrm{~dB}$ for two-port devices for the first prototypes. Recently the concept has been implemented to create the integrated, facet-free Michelson interferometer shown in Fig. 9 [32]. These have also been implemented in on-chip interferometers and precision cavities for mode-locked lasers [16] and provide the precision required for a new generation of precision-controlled coupled-cavity lasers [33].

Pressure to improve energy efficiency and reduce chip real estate motivates higher-density photonic components. Numbers of components have increased from tens of devices in the 1990s to hundreds of devices per chip in the last five years [7], and most recently 1700 components for the latest wavelength multiplexed transmitter chips [34]. This now drives a step change in component-level miniaturization.

\section{NANO-PHOTONIC INTEGRATION}

Smaller components require higher optical confinement than is realistically achievable in substrate-based photonic integration. Therefore a new class of circuits based on membrane technology is emerging. A range of heterogeneous implementations has been proposed. The mostly widely studied
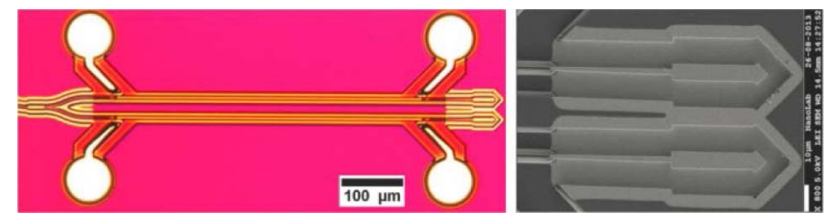

Fig. 9. Facet-free, integrated broadband reflectors (right). Also shown on left: a Michelson interferometer constructed with one-port integrated reflectors [32]. 
approaches focus on the coupling of InP gain regions to silicon-on-insulator waveguide technology, and these are discussed elsewhere in this Special Issue. In this section, we focus on the IMOS technique, which enables all the photonic devices to be implemented within the same monolithic InP integrated photonic membrane, without any critical optical couplings or dependencies on other separately produced photonic wafers. Active and passive components are integrated within a single InP photonic membrane without introducing assembly level performance variability or assembly level dependencies. We review recent innovations in this IMOS technology.

\section{A. Microrings}

High-optical-confinement waveguides enable some of the smallest components for photonic processing. Microrings are of particular interest in the creation of high- $Q$ resonators, but here there is a need to resolve precision features in directional couplers as shown in Fig. 10. Appropriately prepared e-beam lithography resists are enabling step changes in pattern definition accuracy [35]. Importantly, this also enables a reduction in sidewall roughness. The methods have so far been studied for InP membrane devices, where the optical waveguide cross sections are $400 \mathrm{~nm} \times 250 \mathrm{~nm}$. Straight waveguide losses had previously ranged from 10 to $15 \mathrm{~dB} / \mathrm{cm}$, but the optimizations with higher precision photoresists have now enabled sidewall roughness reduction and loss reduction to a best case value of $3.3 \mathrm{~dB} / \mathrm{cm}$ [35], approaching the values currently quoted for foundrysourced, undoped $500 \mathrm{~nm} \times 210 \mathrm{~nm}$ silicon-on-insulator waveguides [36].

\section{B. Optical Modulators}

Enhanced modulation efficiency is achieved by enhancing the optical overlap with the phase modulated active layer. Industry-produced InP modulators already achieve modulation efficiencies of down to $3.5 \mathrm{~V}_{\pi} \mathrm{mm}$ [8]. Further improvements are feasible with the very high optical overlap achieved in substrate-removed devices: values as low as $0.6 \mathrm{~V}_{\pi} \mathrm{mm}$ have now been reported under push-pull drive $[37,38]$. Such substrate-removal techniques also open the way to low electronic parasitics. Longer devices have enabled bandwidth demonstrations to $67 \mathrm{GHz}$.

Photonic membrane devices are typically less than $1 \mu \mathrm{m}$ in thickness, and a key challenge for efficient ultra-highconfinement optoelectronic components is the creation of low resistance electrodes with low optical loss. Ag/Ge-based



Fig. 10. Precision directional coupler for fabricating microrings in the IMOS platform [35]. ohmic contacts have been developed for n-type InP with both low contact resistances and relatively low optical losses. A specific contact resistance as low as $1.5 \times 10^{6} \Omega \mathrm{cm}^{2}$ is achieved by optimizing the Ge layer thickness and annealing conditions [39]. The use of Ge as the first deposited layer results in a low optical absorption loss in the telecommunication wavelength range. Compared to Au-based contacts, the Ag-based metallization also shows a considerably reduced spiking effect in the direction of the active layer after annealing. A factor of 5 reduction of the propagation loss compared to the conventional $\mathrm{Au} / \mathrm{Ge} / \mathrm{Ni}$ contact is experimentally demonstrated. Further optimizations are anticipated with lower optical loss, lower optical resistance contacting techniques.

\section{Membrane Lasers}

The first electrically pumped IMOS laser has recently been demonstrated using an SOA integrated within a passive ring cavity and MMI out-coupler, all within one InP membrane [40]. The amplifier design consists of two vertically stacked waveguiding layers: a $250 \mathrm{~nm}$ thick, Q1.58 active layer vertically coupled with a $300 \mathrm{~nm}$ passive layer. The n-contact layers are $100 \mathrm{~nm}$ thick and are formed by an $\mathrm{n}^{+}$-doped Q1.25 quaternary layer and an n-doped InP layer, which are sandwiched between the active and passive waveguiding layers. The p-contact layers consist of a p-doped InP cladding layer, a p-contact region formed by quaternary spacer layers, and highly $\mathrm{p}^{+}$-doped InGaAs. The entire layer stack is bonded to $\mathrm{SiO}_{2} / \mathrm{Si}$ carrier wafer using $\mathrm{BCB}$ as the bonding material. Figure 11 shows the completed laser.

The width and length of the amplifier section are $700 \mathrm{~nm}$ and $300 \mu \mathrm{m}$, respectively. The optical power is coupled between the active amplifier and passive circuit elements with a short $10 \mu \mathrm{m}$ length taper. The total ring cavity length is $2 \mathrm{~mm}$, and the laser output is coupled through a $2 \times 2$ MMI coupler with a 50/50 power splitting ratio. Light is coupled from the passive waveguides to optical fiber with a diffraction grating coupler. The SOA diode characteristic shows a $15 \Omega$ forward series resistance and only $20 \mathrm{nA}$ dark current at $-3 \mathrm{~V}$ reverse bias. The pulsed light-current characteristics of the laser indicate a threshold current of $200 \mathrm{~mA}$ with a peak fiber-coupled power of $>100 \mu \mathrm{W}$. Further optimizations of the cavity,

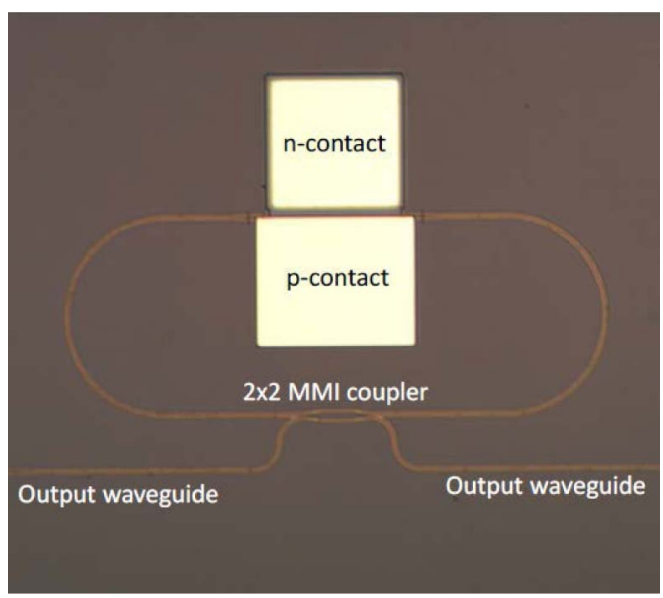

Fig. 11. Ring laser in an active-passive IMOS [40]. 
contacts, and out-coupler gratings may be expected to increase the efficiency and power.

\section{Surface Gratings}

Optical connections to high-confinement membrane technologies require considerable mode-size adaptations, motivating research into surface grating techniques that match the highconfinement nano-wires to a mode size that matches, for example, $9 \mu \mathrm{m}$ fiber cores. Surface gratings have typically suffered from the optical properties of the underlying layers, introducing unnecessary sensitivity to variations in the fabrication process flow. We have recently demonstrated a metal grating coupler compatible with the membrane photonic approach. This consists of a buried metal grating and a metal mirror that is insensitive to the underlying substrate. In contrast to dielectric gratings, simulations predict strongly reduced parasitic leakage of light to the substrate and performance that is independent of the optical buffer thickness. A nonapodized design has been proposed with a theoretical fiber-to-chip coupling efficiency at $1.55 \mu \mathrm{m}$ of up to $73 \%$, and apodized designs are expected to show 89\% coupling efficiencies, with $3 \mathrm{~dB}$ bandwidth of 61 and $78 \mathrm{~nm}$, respectively [41].

Figure 12 shows an SEM cross section through the metal grating [42], highlighting the InP membrane waveguide structure and the grating that is implemented with patterned $\mathrm{SiO}_{x}$ and Ag overlay. The grating is designed to couple light with a wavelength of $1.55 \mu \mathrm{m}$ to a single-mode fiber (SMF) with $9 \mu \mathrm{m}$ core diameter. The grating has a periodicity of $635 \mathrm{~nm}$, a $50 \%$ filling factor, and depth of $125 \mathrm{~nm}$ in the $\mathrm{SiO}_{x}$ layer. The InP waveguiding layer has a thickness of $300 \mathrm{~nm}$ and a footprint of $16 \mu \mathrm{m} \times 25 \mu \mathrm{m}$ in the metal grating area. The grating coupler is connected to a $400 \mathrm{~nm}$ wide waveguide via an adiabatic taper with a length of $250 \mu \mathrm{m}$. Recent experimental data show a $3 \mathrm{~dB}$ bandwidth of $61 \mathrm{~nm}$ and chip-to-fiber coupling efficiency of $54 \%$ for the nonapodized couplers.

\section{OUTLOOK}

InP integrated photonics has offered premium solutions for telecommunications infrastructure, but it is increasingly clear that the technology is poised to impact a much broader range of market segments. Many of the circuit techniques and components are readily reconfigured to enable high-performance photonic solutions in sensing, imaging, and high-speed signal analysis. Integrating the wide range of photonic functions is now robustly demonstrated. Further innovation is now

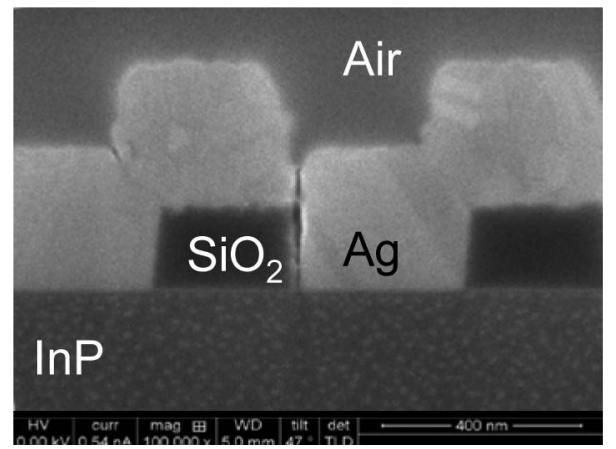

Fig. 12. Cross section through a membrane metallic grating [42]. anticipated to reduce assembly complexity and to simplify design and test.

PICs commonly require combination with interposers, electronics, and other integrated optical and micro-optical systems. Further integration is required at a number of levels from hybrid schemes for system in package integration through to the semiconductor technology level heterogeneous integration. We distinguish as follows:

- Hybrid approaches combine the processed chips in a package and/or on an interposer.

- Heterogeneous approaches combine different materials on a single substrate during the process.

The term heterogeneous integration therefore spans from the use of InP active areas that may power silicon photonics to the full platform capability targeted here within the IMOS platform. The IMOS approach offers compelling advantages, with the full PIC being created in one monolithic crystal. The membrane waveguide concept provides ultra-high confinement for circuit-level miniaturization, and a wafer-scale processing methodology that is poised to enable simpler chip level connections.

Photonic-electronic convergence is a logical next step for the IMOS platform: thin III-V photonic membrane layers could just as easily be placed on top of silicon electronic wafers as on the $\mathrm{Si} / \mathrm{SiO}_{2}$ wafers currently in use. Figure 13 shows the concept for an InP integrated photonic membrane containing lasers, modulators, detectors, and a range of other passive waveguiding devices.

Convergence of InP with dielectric-waveguide technologies [43] offers a compelling new hybrid integration platform as well with potential advantages in relation to single-material monolithic approaches. It adds the versatility of optical devices such as lasers, high-speed modulators, amplifiers, or photodiodes on InP to the low propagation loss of the low-confinement waveguides in (for example) TriPleX technology. The platform allows for high-density and high-portcount coupling between both material platforms, and the low-loss optical fiber matched spot-size converters in TriPleX [44,45]. An example implementation is shown schematically in Fig. 14, with fiber-optic connections from the left connecting to an InP PIC on the right via a TriPleX optical interposer.

Scaling laws for InP integrated photonics are similar to other thin-film fabrication technologies such as CMOS electronics or silicon photonics [8], so with increased wafer size

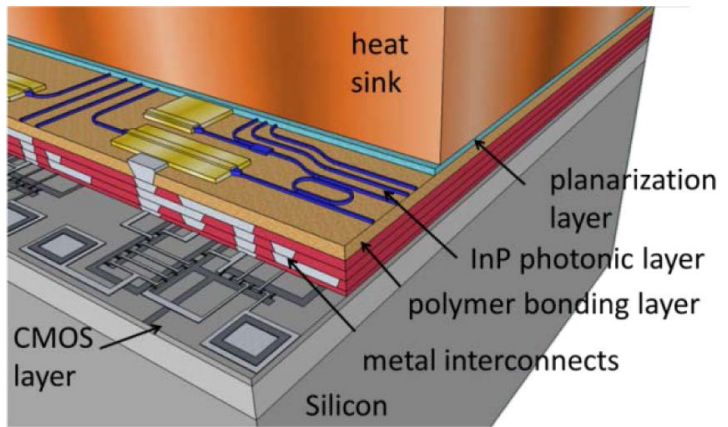

Fig. 13. Photonic membrane layer on top of silicon electronics, simplifying electronic photonic connection and assembly technology at the wafer scale. 


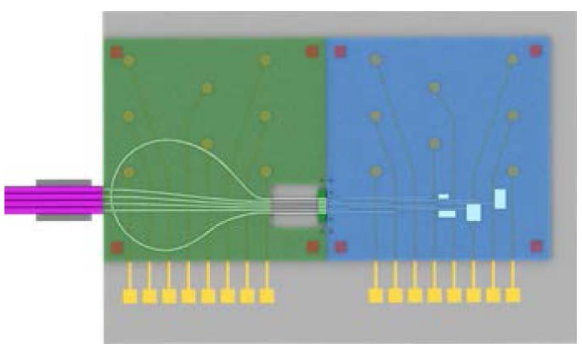

Fig. 14. Low-loss dielectric-waveguide technology combined with powerful InP photonic integrated circuit technology for automated optical alignment [44].

and fab throughput, the square millimeter price is expected to decrease strongly. This has been the driving force behind the photonics foundry model initiative [46]. As square millimeter costs are strongly dependent on the total volume, fab customers share the fixed production costs, enabling economies of scale across market segments. This sharing of technology costs across many application fields reduces costs even for small users. The development of advanced PDKs and design libraries will play an increasing role in reducing design and test complexity as well.

Integrated photonic technologies in combination with high-performance electronics, innovative connection technologies, and economies of scale have the potential to make game-changing performance advances. Disruptive products are anticipated in a wide range of sectors from precision metrology, environmental monitoring, and safety systems to predictive healthcare, data center connectivity, and computer communications.

\section{Acknowledgment}

The authors acknowledge the EC FP7 project Paradigm, the Dutch Memphis project, and the many partners within the projects who have played such an important part in developing the generic integration methodology. Many of the circuits reported in this work have been supplied via the JePPIX platform, and a number of circuits have also been produced by researchers at COBRA.

\section{REFERENCES}

1. C. R. Doerr and K. Okamoto, "Planar lightwave circuits in fiberoptic communications," in Optical Fiber Telecommunications $V A$ : Components and Subsystems, I. P. Kaminow, T. Li, and A. E. Willner, eds., 5th ed. (Academic, 2008), pp. 269-342.

2. R. A. Griffin, S. K. Jones, N. Whitbread, S. C. Heck, and L. N. Langley, "InP Mach-Zehnder modulator platform for 10/40/ 100/200-Gb/s operation," IEEE J. Sel. Top. Quantum Electron. 19, 158-166 (2013).

3. R. Stabile, A. Rohit, and K. A. Williams, "Monolithically integrated $8 \times 8$ space and wavelength selective cross-connect," J. Lightwave Technol. 32, 201-207 (2014).

4. F. Kish, R. Nagarajan, D. Welch, P. Evans, J. Rossi, J. Pleumeekers, A. Dentai, M. Kato, S. Corzine, R. Muthiah, M. Ziari, R. Schneider, M. Reffle, T. Butrie, D. Lambert, M. Missey, V. Lal, M. Fisher, S. Murthy, R. Salvatore, S. Demars, A. James, and C. Joyner, "From visible light-emitting diodes to large scale III-V photonic integrated circuits," Proc. IEEE 1010, 2255-2270 (2013).

5. R. Nagarajan, C. R. Doerr, and F. A. Kish, "Semiconductor photonic integrated circuit transmitters and receivers," in Optical Fiber Telecommunications, I. Kaminow, T. Li, and A. Wilner, eds. (Elsevier, 2013), Chap. 2, Vol. VIA, pp. 25-98.
6. M. K. Smit, X. Leijtens, H. Ambrosius, E. Bente, J. van der Tol, E. Smalbrugge, T. de Vries, E. J. Geluk, J. Bolk, R. van Veldhoven, L. Augustin, P. Thijs, D. d'Agostino, H. Rabbani, K. Ławniczuk, S. Stopinski, S. Tahvili, A. Corradi, E. Kleijn, D. Dzibrou, M. Felicetti, E. Bitincka, V. Moskalenko, J. Zhao, R. Santos, G. Gilardi, W. Yao, K. Williams, R. Stabile, P. Kuindersma, J. Pello, S. Bhat, Y. Jiao, D. Heiss, G. Roelkens, M. Wale, P. Firth, F. Soares, N. Grote, M. Schell, H. Debregeas, M. Achouche, J. L. Gentner, A. Bakker, T. Korthorst, D. Gallagher, A. Dabbs, A. Melloni, F. Morichetti, D. Melati, A. Wonfor, R. Penty, R. Broeke, B. Musk, and D. Robbins, "An introduction to InP-based generic integration technology," Semicond. Sci. Technol. 29, 083001 (2014).

7. M. K. Smit, J. van der Tol, and M. T. Hill, "Moore's law in photonics," Laser Photon. Rev. 6, 1-13 (2012).

8. JePPIX roadmap, 2015, http://www.jeppix.eu/document_store/ JePPIXRoadmap2015.pdf.

9. http://paradigm.jeppix.eu/.

10. http://www.jeppix.eu/.

11. F. Bontempi, S. Faralli, X. J. M. Leijtens, J. Bolk, G. Contestible, and N. Andriolli, "A 40 Gb/s InP-monolithically integrated DPSKdemodulator enhanced by cross-gain-compression in an SOA," Opt. Commun. 340, 155-158 (2015).

12. K. Ławniczuk, C. Kazmierski, J. G. Provost, M. J. Wale, R. Piramidowicz, P. Szczepański, M. K. Smit, and X. J. M. Leijtens, "InP-based photonic multiwavelength transmitter with DBR laser array," IEEE Photon. Technol. Lett. 25, 352-354 (2013).

13. S. Tahvili, S. Latkowski, E. Smalbrugge, X. J. M. Leijtens, P. J. Williams, M. J. Wale, J. Parra-Cetina, R. Maldonado-Basilio, P. Landais, M. K. Smit, and E. A. J. M. Bente, "InP-based integrated optical pulse shaper: demonstration of chirp compensation," IEEE Photon. Technol. Lett. 25, 450-453 (2013).

14. X. Guo, V. Olle, A. Quarterman, A. Wonfor, R. V. Penty, and I. H. White, "Monolithically integrated selectable repetition-rate laser diode source of picosecond optical pulses," Opt. Lett. 39, 4144-4147 (2014).

15. S. Latkowski, V. Moskalenko, S. Tahvili, L. Augustin, M. K. Smit, K. A. Williams, and E. A. J. M. Bente, "Monolithically integrated $2.5 \mathrm{GHz}$ extended cavity mode-locked ring laser with intra-cavity phase modulators," Opt. Lett. 40, 77-80 (2015).

16. C. Gordón, R. Guzmán, X. Leijtens, and G. Carpintero, “On-chip mode-locked laser diode structure using multimode interference reflectors," Photon. Res. 3, 15-18 (2015).

17. V. Moskalenko, S. Latkowski, S. Tahvili, T. de Vries, M. Smit, and E. Bente, "Record bandwidth and sub-picosecond pulses from a monolithically integrated mode-locked quantum well ring laser," Opt. Express 22, 28865-28874 (2014).

18. B. W. Tilma, Y. Jiao, J. Kotani, E. Smalbrugge, H. P. M. M. Ambrosius, P. J. Thijs, X. J. M. Leijtens, R. Notzel, M. K. Smit, and E. A. J. M. Bente, "Integrated tunable quantum dot laser for optical coherence tomography in the $1.7 \mu \mathrm{m}$ wavelength region," IEEE J. Quantum Electron. 48, 87-98 (2012).

19. D. d'Agostino, R. Broeke, M. Boerkamp, J. Mink, H. P. M. M. Ambrosius, and M. K. Smit, "AWG based wavelength-meter with pm resolution," in Proceedings of the 17th European Conference on Integrated Optics and Technical Exhibitionan, 19th Microoptics Conference (ECIO-MOC) (2014), paper Tu2bR2.

20. Q. Cheng, R. Stabile, A. Rohit, A. Wonfor, R. V. Penty, I. H. White, and K. A. Williams, "First demonstration of automated control and assessment of a dynamically reconfigured monolithic $8 \times 8$ wavelength-and-space switch [Invited]," J. Opt. Commun. Netw. 7, A388-A395 (2015).

21. Q. Cheng, A. Wonfor, J. L. Wei, R. V. Penty, and I. H. White, "Monolithic MZI-SOA hybrid switch for low-power and low-penalty operation,” Opt. Lett. 39, 1449-1452 (2014).

22. E. Kleijn, D. Melati, A. Melloni, T. de Vries, M. K. Smit, and X. J. M. Leijtens, "Multimode interference couplers with reduced parasitic reflections," IEEE Photon. Technol. Lett. 26, 408-410 (2014).

23. J. J. G. M. van der Tol, M. Felicetti, and M. K. Smit, "Increasing tolerance in passive integrated optical polarization converters," J. Lightwave Technol. 30, 2884-2889 (2012). 
24. D. O. Dzibrou, J. J. G. M. van der Tol, and M. K. Smit, "Improved fabrication process of low-loss and efficient polarization converters in InP-based photonic integrated circuits," Opt. Lett. 38, 1061-1063 (2013).

25. D. O. Dzibrou, J. J. G. M. van der Tol, and M. K. Smit, "Tolerant polarization converter for InGaAsP-InP photonic integrated circuits," Opt. Lett. 38, 3482-3484 (2013).

26. M. K. Smit, E. C. M. Pennings, and H. Blok, "A normalized approach to the design of optimal optical waveguide bends," J. Lightwave Technol. 11, 1737-1742 (1993).

27. R. Stabile, A. Albores-Mejia, and K. A. Williams, "Monolithic active-passive $16 \times 16$ optoelectronic switch," Opt. Lett. 37, 4666-4668 (2012).

28. R. Stabile and K. A. Williams, "Relaxed dimensional tolerance whispering gallery microbends," J. Lightwave Technol. 29, 1892-1898 (2011).

29. D. d'Agostino, G. Carnicella, C. Ciminelli, H. P. M. M. Ambrosius, and M. K. Smit, "Design of a compact high-performance InP ring resonator," in Third Mediterranean Photonics Conference (2014).

30. E. Kleijn, M. K. Smit, and X. J. M. Leijtens, "New analytical arrayed waveguide grating model," J. Lightwave Technol. 31, 3309-3314 (2013).

31. E. Kleijn, M. K. Smit, and X. J. M. Leijtens, "Multimode interference reflectors: a new class of components for photonic integrated circuits," J. Lightwave Technol. 31, 3055-3063 (2013).

32. S. Stopinski, M. Malinowski, R. Piramidowicz, C. Kazmierski, M. K. Smit, and X. J. M. Leijtens, "Photonic integrated multichannel WDM modulators for data read-out units," J. Lightwave Technol. 32, 4481-4489 (2014).

33. D. d'Agostino, D. Lenstra, H. P. M. M. Ambrosius, and M. K. Smit, "Coupled cavity laser based on anti-resonant imaging via multimode interference," Opt. Lett. 40, 653-656 (2015).

34. J. Summers, T. Vallaitis, P. Evans, M. Ziari, P. Studenkov, M. Fisher, J. Sena, A. James, S. Corzine, D. Pavinski, J. Ou-Yang, M. Missey, D. Gold, W. Williams, M. Lai, D. Welch, and F. Kish, "Monolithic InP-based coherent transmitter photonic integrated circuit with $2.25 \mathrm{Tbit} / \mathrm{s}$ capacity," Electron. Lett. 50, 1150-1152 (2014).
35. Y. Jiao, J. Pello, A. Millan Mejia, L. Shen, E. Smalbrugge, E. J. Geluk, M. Smit, and J. van der Tol, "Fullerene-assisted electron-beam lithography for pattern improvement and loss reduction in InP membrane waveguide devices," Opt. Lett. 39, 1645-1648 (2014).

36. http://www.epixfab.eu/.

37. S. Dogru and N. Dagli, "0.77-V drive voltage electro-optic modulator with bandwidth exceeding $67 \mathrm{GHz}$," Opt. Lett. 39, 6074-6076 (2014).

38. S. Dogru and N. Dagli, " $0.2 \mathrm{~V}$ drive voltage substrate removed electro-optic Mach-Zehnder modulators with MQW cores at $1.55 \mu \mathrm{m}$," J. Lightwave Technol. 32, 435-439 (2014).

39. L. Shen, V. Dolores-Calzadilla, C. W. H. A. Wullems, Y. Jiao, A. Millan-Mejia, A. Higuera-Rodriguez, D. Heiss, J. J. G. M. van der Tol, H. P. M. M. Ambrosius, G. Roelkens, and M. K. Smit, "Lowoptical-loss, low-resistance $\mathrm{Ag} / \mathrm{Ge}$ based ohmic contacts to n-type InP for membrane based waveguide devices," Opt. Mater. Express 5, 393-398 (2015).

40. Y. Jiao, D. Heiss, L. Shen, S. Bhat, M. Smit, and J. van der Tol, "First demonstration of an electrically pumped laser in the InP membrane on silicon platform," in Proceedings Integrated Photonics Research (2015).

41. V. M. Dolores-Calzadilla, D. Heiss, and M. K. Smit, "Highly efficient metal grating coupler for membrane-based integrated photonics," Opt. Lett. 39, 2786-2789 (2014).

42. A. Higuera-Rodriguez, V. Dolores-Calzadilla, Y. Jiao, E. J. Geluk, D. Heiss, and M. K. Smit, "High efficiency metallic grating couplers for membranes on photonic integrated circuits," Opt. Lett. 40, 2755-2757 (2015).

43. K. Wörhoff, R. G. Heideman, A. Leinse, and M. Hoekman, "TriPleX: a versatile dielectric photonic platform," Adv. Opt. Technol. 4, 189-207 (2015).

44. http://www.phastflex.eu/.

45. Photonics West 2015 exhibition, Moscone Center in San Francisco, USA (2015).

46. M. K. Smit, X. J. M. Leijtens, E. A. J. M. Bente, J. J. G. M. van der Tol, H. P. M. M. Ambrosius, D. J. Robbins, M. J. Wale, N. Grote, and M. Schell, "Generic foundry model for InP-based photonics," IET Optoelectron. 5, 187-194 (2011). 